\title{
Complementary Filter Design Based Error Allocation for Attitude Estimation with Low-Cost Sensor
}

\author{
Longhe Yang ${ }^{1}$, Jianxin Ren ${ }^{1, *}$, Kaiyue Song ${ }^{1}$, Xiaofei $\mathrm{Ma}^{2}$ and Xinghui Yang ${ }^{3}$ \\ ${ }^{1}$ School of Automation Northwestern Polytechnical University Xi'an 710000, P. R. China \\ ${ }^{2}$ Northern Information Control Research Institute Group Co., Ltd Nanjing 210000, P.R. China \\ ${ }^{3}$ Beijing Automation Control Equipment Research Institute Beijing 100074, P. R. China \\ ${ }^{*}$ Corresponding author
}

\begin{abstract}
This paper investigates complementary filter designed for attitude estimation for low-cost inertial measurement unit and magnetometer. An error allocation strategy which focuses on two weight parameter matrices regulated according to output modulus of accelerometer and magnetometer automatically, is proposed. The strategy of error allocation fuses gravity reference error angle comes from accelerometer and geomagnetic reference error angle comes from magnetometer to get the reference angle error which is used to correct the angular velocity drift of gyroscope after passed proportion and integral estimator (PI estimator). Attitude is obtained with quaternion solution. Experimental results show that this error allocation strategy can get reference angle error when output modulus of accelerometer is under the acceleration threshold or the changing horizontal magnetic component is below the changing threshold.
\end{abstract} filter

Keywords-attitude estimation; error allocation; complementary

\section{INTRODUCTION}

Attitude estimation of aero craft is an important part for control and measure using low-cost inertial measurement unit and magnetometer. With the growing application demands for aerocraft, it is urgent to develop robust and low computa- tional complexity for attitude estimation with low-cost inertial measurement unit and magnetometer [1-2]. Many researchers focused on different filters such as frequency domain linear filter (LP, low-pass filter, etc), kalman filter (KF) or particle filter (PF) to estimate attitude. In [3-5], frequency domain linear filter is used to estimate attitude. In [6-7], Extended Kalman Filter (EKF) is widely applied to aerospace. Particle filter is used for special attitude estimation application with multi information fusion [8]. Filters as KF, EKF, PF and LP, are all facing high computational complexity and model dependence that the system cannot satisfy calculation speed with single processor (STM32F103,etc) to deal with multiple task at once. sensor characteristics of gyro and accelerometer to Linear complementary filter is proposed to fuse data of gyroscope deal with high-pass filter for angular velocity and data of accelerometers deal with low-pass filter for acceleration in [9]. Linear complementary filter fuses sensor characteristics in frequency domain and its computational complexity is much lower than the kalman filter and particle filter, and it is hard to design suitable cut-off frequency for low-pass filter and highpass filter. Similar to Linear complementary filter, Nonlinear complementary filter can also provide fusing strategy for gyroscope and accelerometer. In [2-3],[6-13], Nonlinear complementary filter is designed by referring to reference physical quantity of geomagnetic or local gravity, then passed by proportion and integral estimator, compensates angular velocity last. Nonlinear complementary filter is usually used when angular motion is stable and linear acceleration is small enough, or provided wrong angular velocity compensation otherwise [13-15].

In this paper, we provide an error allocation strategy to make full use of reference error angle which comes from local gravity and geomagnetic. The reference error angle consists of two parts, gravity reference error angle and geomagnetic reference error angle. When aerocraft acceleration is zero, the gravity reference error angle is believable and the reference error angle is only from attitude error. While aerocraft acceleration is not zero and the reference error angle is from attitude error and aerocraft acceleration which we try to eliminate. So modulus of aerocraft acceleration must be small value before getting gravity reference error angle. Geomagnetic reference error angle can be used when there does not exist magnetic anomaly in local. According to the basis characteristics of gravity reference error angle and geomagnetic reference error angle, the paper design two allocation matrices to strength- en heading angle compensation with geomagnetic reference error angle and horizontal attitude angle compensation with gravity.

The body of this paper consists of five sections. Section II gives notation definition, measures used and tell the difference between linear complementary filter and nonlinear complementary filter. Section III gives the error allocation for complementary filter design and explains its physical principle. Section IV verified that the error allocation for complementary filter is effective. All work in this paper is summarized in section $\mathrm{V}$.

\section{PRoblem Formulation And Notation}

\section{A. Notation and Reference Physical Information}

Coordinate frames used in this paper listed in the following,

The navigation frame (n-frame), is used to which is selected local geographic frame whose $X$ axis, $Y$ axis and $Z$ axis point to the directions of east ( $E$ ), north $(N)$ and the local up vertical $(U)$. Mapping relation can be expressed, 


$$
(X, Y, Z) \rightarrow(E, N, U)
$$

The body frame (b-frame), is an orthogonal axis set which is used to represent pitch angle $(\theta)$, roll angle $\left({ }^{\gamma}\right)$, heading angle $\left({ }^{\psi}\right.$ ) with $x$ axis, ${ }^{y}$ axis and $z$ axis, and point to right, forward and up. Mapping relation can be expressed,

$$
(x, y, z) \rightarrow(\theta, \gamma, \psi)
$$

Notation description used in this paper listed in the following,

Direction cosine matrix (DCM), $\mathbf{C}_{n}^{b}$ is orthogonal matrix or need orthogonal processing after each updating, and used for conversion between n-frame and b-frame.

Attitude quaternion vector $\hat{\mathbf{q}}=\left[\begin{array}{llll}q_{0} & q_{1} & q_{2} & q_{3}\end{array}\right]^{T}$ consists of four elements, and $\hat{\mathbf{q}}$ is usually normalized. The normalized $\hat{\mathbf{q}}$ and DCM after orthogonal processing are equivalent refer to attitude solution.

Mathematical operations need to be clearly pointed out. For quaternion product “ $\otimes$ ”, its operational rule can refer to [15]. For vector product “ $\times$ ”, its operational rule can refer to [15].

$$
\begin{aligned}
& \text { For vector, } \mathbf{v}=\left[\begin{array}{lll}
v_{x} & v_{y} & v_{z}
\end{array}\right]^{T} \text {, its 2-norm of vector } \mathbf{v} \\
& \|\mathbf{v}\|=\sqrt{v_{x}^{2}+v_{y}^{2}+v_{z}^{2}}
\end{aligned}
$$

Reference physical information in this paper is local geomagnetic vector $\mathbf{m}^{n}$ and local gravity vector $\mathbf{g}^{n}, \mathbf{m}^{n}$ has constant horizontal magnetic component and constant vertical magnetic component in local. ${ }^{n}$ has only vertical component. Reference vectors $\mathbf{m}^{n}$ and $\mathbf{g}^{n}$ are described as,

$$
\mathbf{m}^{n}=\left[\begin{array}{lll}
0 & b_{y} & b_{z}
\end{array}\right]^{T}, \mathbf{g}^{n}=\left[\begin{array}{lll}
0 & 0 & g
\end{array}\right]^{T}
$$

where

$$
\mathbf{H}=\left[h_{x}, h_{y}, h_{z}\right]^{T}, \quad b_{y}=\sqrt{h_{x}^{2}+h_{y}^{2}}, \quad b_{z}=h_{z}, \quad \mathbf{H}=\mathbf{C}_{b}^{n} \mathbf{m}^{b}
$$
frame.

$\mathbf{C}_{b}^{n}$ is attitude matrix, $\mathbf{m}^{b}$ is magnetometer output in b-

\section{B. Measurements}

The measurements available from a typical inertial measurement unit are 3 axis rate gyroscopes, 3 axis accelerometers and 3 axis magnetometers. MEMS inertial measurement unit is selected. As angular velocity error compensation is the final purpose using complementary filter, its error analysis is necessary. Accelerometers are used to get velocity by measuring linear acceleration.

Ignoring scale factor error, error model of angular velocity used in this paper is

$$
\begin{aligned}
& \hat{\boldsymbol{\omega}}=\boldsymbol{\omega}+\delta \boldsymbol{\omega} \\
& \delta \boldsymbol{\omega}=\mathbf{b}_{0}+\boldsymbol{\varepsilon}_{0}+\boldsymbol{\varepsilon}_{g} \\
& \dot{\boldsymbol{\varepsilon}}_{0}=-1 / \tau \cdot \boldsymbol{\varepsilon}_{0}+\boldsymbol{\varepsilon}_{g 1}
\end{aligned}
$$

where $\hat{\boldsymbol{\omega}}=\left[\begin{array}{lll}\hat{\omega}_{x} & \hat{\omega}_{y} & \hat{\omega}_{z}\end{array}\right]^{T}$ is the actual angular velocity of rate gyroscopes, $\boldsymbol{\omega}=\left[\begin{array}{lll}\omega_{x} & \omega_{y} & \omega_{z}\end{array}\right]^{T}$ is the ideal angular velocity of rate gyroscopes. $\delta \boldsymbol{\omega}=\left[\begin{array}{lll}\delta \omega_{x} & \delta \omega_{y} & \delta \omega_{z}\end{array}\right]^{T}$ is total error angular velocity including constant bias $\mathbf{b}_{0}=\left[\begin{array}{lll}b_{0, x} & b_{0, y} & b_{0, z}\end{array}\right]^{T}$, first order Markov $\boldsymbol{\varepsilon}_{0}$ and other unknown error $\boldsymbol{\varepsilon}_{g}$ and $\boldsymbol{\varepsilon}_{g 1}$. We take $\boldsymbol{\varepsilon}_{g}$ and $\boldsymbol{\varepsilon}_{g 1}$ as the zero mean white Gaussian noise in this paper, $\tau$ is time correlation constant. PI estimator will be used to deal with constant bias $\mathbf{b}_{0}$ and slowly varying error $\boldsymbol{\varepsilon}_{0}$, where proportional term used to regulate slowly varying error $\boldsymbol{\varepsilon}_{0}$ and integral term used to regulate constant bias $\mathbf{b}_{0}$.

After correction to scale factor and bias, output of magnetometer is expressed as

$$
\hat{\mathbf{m}}^{b}=\mathbf{m}^{b}
$$

where $\hat{\mathbf{m}}^{b}=\left[\begin{array}{lll}\hat{m}_{x}^{b} & \hat{m}_{y}^{b} & \hat{m}_{z}^{b}\end{array}\right]^{T}$ is actual magnetic field intensity of magnetometers' output in b-frame, $\mathbf{m}^{b}$ is the ideal magnetic field intensity.

For accelerometers, the sensitive physical quantities contain aerocraft acceleration and gravity acceleration. Aerocraft acceleration is the direct physical quantity acquiring velocity, while gravity acceleration is the reference physical quantity used in the work. Relation between two physical quantity is

$$
\mathbf{f}^{b}=\mathbf{a}^{b}+\mathbf{g}^{b}
$$

Where $\mathbf{f}^{b}=\left[\begin{array}{lll}f_{x}^{b} & f_{y}^{b} & f_{z}^{b}\end{array}\right]^{T}$ is the output of accelerometers, $\mathbf{a}^{b}$ is aerocraft acceleration, $\mathbf{g}^{b}=\mathbf{C}_{n}^{b} \cdot \mathbf{g}^{n}$ is gravity acceleration, $\mathbf{g}^{n}=\left[\begin{array}{lll}0 & 0 & g\end{array}\right]^{T}$ is reference gravity and $g \approx 9.8\left(\mathrm{~m} / \mathrm{s}^{2}\right)$.

\section{Complementary Filter}

The complementary filter design in this paper is based on Mahony complementary filter proposed by Robert Mahony in [2-3], [6].

Attitude estimator using complementary filter is 


$$
\begin{aligned}
& \dot{\hat{\mathbf{q}}}=\hat{\mathbf{q}} \otimes \overline{\boldsymbol{\omega}} \\
& \delta \boldsymbol{\omega}=k_{p} \mathbf{e}+k_{I} \int_{t} \mathbf{e} d t \\
& \mathbf{e}=\mathbf{D}_{\text {yaw }} \cdot \boldsymbol{\varepsilon}_{\text {mag }}+\mathbf{D}_{h} \cdot \boldsymbol{\varepsilon}_{a c c}
\end{aligned}
$$

where $\hat{\mathbf{q}}=\left[\begin{array}{llll}q_{0} & q_{1} & q_{2} & q_{3}\end{array}\right]^{T}$ is attitude quaternion vector, $\overline{\boldsymbol{\omega}}=\left[0, \hat{\boldsymbol{\omega}}^{T}-\delta \boldsymbol{\omega}^{T}\right]^{T}$ is angular velocity quaternion vector, $\delta \boldsymbol{\omega}$ is estimated error by PI estimator, $\mathbf{e}=\left[\begin{array}{lll}e_{x} & e_{y} & e_{z}\end{array}\right]^{T}$ is angle error vector named reference angle error, $\boldsymbol{\varepsilon}_{\text {mag }}$ is the geomagnetic reference error angle, ${ }^{\boldsymbol{\varepsilon}_{\text {acc }}}$ is the gravity reference error angle, $k_{p}$ is constant proportion parameter and $k_{I}$ is constant integral parameter, $\mathbf{D}_{\text {yaw }}=\operatorname{diag}\left(\left[\alpha_{x}, \alpha_{y}, \alpha_{z}\right]\right)$ is the heading error allocation matrix and $\mathbf{D}_{h}=\operatorname{diag}\left(\left[\beta_{x}, \beta_{y}, \beta_{z}\right]\right)$ is the horizontal attitude error allocation matrix.

Equation (4) explains the relationship of sensor data between accelerometer and gyroscope. Traditional linear complementary filter fuses sensor characteristics of gyroscope and accelerometer according to the fact that data of gyroscope

providing high precision for high dynamic motion in short time and data of accelerometer has long term stable precision for low dynamic motion. However, angular velocity drift exists in the angular velocity output of gyro and overly sensitive exists in the acceleration. High-pass filter designed for angular velocity output of gyroscope can suppress angular velocity drift, designed low-pass filter for acceleration data. Then combining both linear filter, traditional linear complementary filter is designed.

Complementary filter designed in this paper still fuses output data of gyro and accelerometer as the traditional linear complementary. But the strategy is different from traditional linear complementary filter, as it uses the earth gravity as reference information and the geomagnetic as reference information to compensate data of gyroscope. The whole compensation process can be seen in "Fig.1".

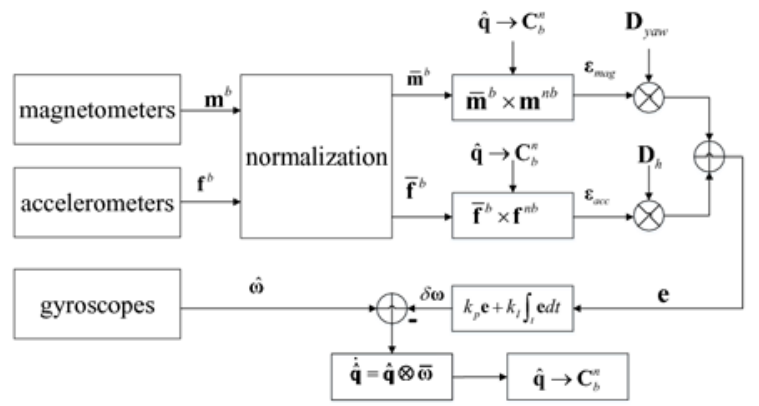

FIGURE I. COMPLEMENTARY FILTER PROCEDURE BASED ERROR ALLOCATION
In fig1, $\overline{\mathbf{m}}^{b}=\mathbf{m}^{b} /\left\|\mathbf{m}^{b}\right\|$ is the normalized magnetometer vector in b-frame, $\mathbf{m}^{n b}=\mathbf{C}_{n}^{b} \mathbf{m}^{n}$ is the reference magnetic vector in b-frame, and $\mathbf{m}^{n}=\left[\begin{array}{lll}0 & b_{y} & b_{z}\end{array}\right]^{T}$ is reference magnetic vector in n-frame, $\boldsymbol{\varepsilon}_{\text {mag }}=\overline{\mathbf{m}}^{b} \times \mathbf{m}^{n b} \quad \overline{\mathbf{f}}^{b}=\mathbf{f}^{b} /\left\|\mathbf{f}^{b}\right\|$ is normalized acceleration provided by accelerometer, $\mathbf{f}^{n b}=\mathbf{C}_{n}^{b} \mathbf{f}^{n}$ is reference acceleration with attitude error, $\mathbf{f}^{n}=\mathbf{g}^{n} / g=\left[\begin{array}{lll}0 & 0 & 1\end{array}\right]^{T}$ is normalized gravity acceleration, $\boldsymbol{\varepsilon}_{a c c}=\overline{\mathbf{f}}^{b} \times \mathbf{f}^{n b} \hat{\mathbf{q}} \rightarrow \mathbf{C}_{b}^{n}$ is the mapping relation between quaternion and DCM.

\section{ERROR ALLOCATION FOR COMPLEMENTARY FILTER DESIGN}

\section{A. Physical Principle of Error Allocation}

Physical explanation to error allocation can be expressed that the geomagnetic reference error angle mapping to heading

angle and the gravity reference error angle mapping to the horizontal attitude angle.

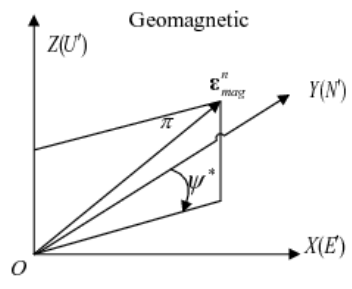

(a)

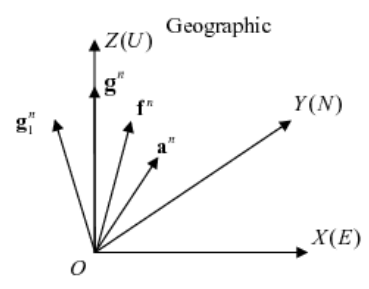

(b)
FIGURE II. PHYSICAL EXPLANATION TO ERROR ALLOCATION

Geomagnetic reference error angle mapping to heading angle can be analyzed in "Fig.2(a)". The mapping relationship is described in the next paragraph.

For convenience, we analyze error allocation for geomagnetic reference error angle in $n$-frame rather than in bframe. In other words, we analyze $\boldsymbol{\varepsilon}_{\text {mag }}^{n}$ in n-frame rather $\boldsymbol{\varepsilon}_{\text {mag }}$ in b-frame. This change does not affect the allocation strategy of $\boldsymbol{\varepsilon}_{\text {mag }}$.

In "Fig.2(a)", $b_{y}$ is the horizontal component of magnetic field and its positive direction coincides with $Y$ axis in magnetic north, $b_{z}$ is the vertical component of magnetic field

and its positive direction coincides with $Z$ axis in magnetic up.

After compensated, data output of magnetometer is assumed to no time-varying error for attitude. If no attitude error $\overline{\mathbf{m}}^{b}$ and $\mathbf{m}^{n b}$ are equivalent which means have the same direction. Then,

$$
\boldsymbol{\varepsilon}_{\text {mag }}=\left\|\overline{\mathbf{m}}^{b}\right\| \cdot\left\|\mathbf{m}^{n b}\right\| \cdot \sin (0)=0
$$


But there exist attitude error in DCM $\mathbf{C}_{n}^{b}$, making angle error vector $\boldsymbol{\varepsilon}_{\text {mag }}^{n}$.

Considering the fact that the restructure reference physical quantity for magnetometer is not one reference axis but one reference surface which consists of $Y$ axis in magnetic north and $Z$ axis in magnetic up, the heading component of $\boldsymbol{\varepsilon}_{\text {mag }}^{n}, \psi^{*}$ is the most significant. While the horizontal component of $\boldsymbol{\varepsilon}_{\text {mag }}^{n}$ should be used carefully.

So the error allocation for geomagnetic reference error angle, should take more important in heading component rather put them as the same important. As angular velocity drift of gyro in $\mathrm{z}$ axis has more influence to the heading error, this error allocation strategy indirectly separates the errors in angular velocity drift of gyro in $Z$ axis.

Gravity reference error angle mapping to the horizontal attitude angle can be analyzed in "Fig.2(b)". The mapping relationship is described in the next paragraph.

In "Fig.2(b)", the relationship between physical quantities in n-frame is

$$
\begin{aligned}
& \mathbf{f}^{n}=\mathbf{a}^{n}+\mathbf{g}^{n} \\
& \mathbf{g}_{1}^{n}=\mathbf{C}_{b}^{n} \mathbf{g}^{b}
\end{aligned}
$$

where DCM $\mathbf{C}_{b}^{n}$ is not ideal attitude matrix, $\mathbf{g}^{n} \neq \mathbf{g}_{1}^{n}$.

For convenience, we set $\mathbf{a}^{n}=\left[\begin{array}{lll}0 & 0 & 0\end{array}\right]^{T}$. Then attitude error between $\mathbf{g}_{1}^{n}$ and $\mathbf{g}^{n}$ mainly come from attitude error in $\mathbf{C}_{b}^{n}$.

Considering the fact that reference information are only one axis and its positive direction coincides with $\mathbf{g}^{n}$ which means the horizontal attitude angle is believable and heading attitude angle is unbelievable. As angular velocity drift of gyro in $x$ axis and ${ }^{y}$ axis has more influence to the horizontal attitude angle error, this error allocation strategy indirectly separates the errors in angular velocity drift of gyro in ${ }^{x}$ axis and ${ }^{y}$ axis.

$$
\text { If } \mathbf{a}^{n} \neq\left[\begin{array}{lll}
0 & 0 & 0
\end{array}\right]^{T}, \boldsymbol{\varepsilon}_{\text {acc }} \text { is divided into two parts, }
$$

$$
\boldsymbol{\varepsilon}_{a c c}=\boldsymbol{\varepsilon}_{d}+\boldsymbol{\varepsilon}_{a c c}^{\prime}
$$

Where $\boldsymbol{\varepsilon}_{d}=\mathbf{a}^{b} \times \mathbf{f}^{n b} /\left\|\mathbf{f}^{b}\right\|$ is part of gravity reference error angle originates in physical movement, $\boldsymbol{\varepsilon}_{a c c}^{\prime}=\mathbf{g}^{b} \times \mathbf{f}^{n b} /\left\|\mathbf{f}^{b}\right\|$ is part of gravity reference error angle originates in attitude error caused by device error. $\boldsymbol{\varepsilon}_{a c c}^{\prime}$ is the error that we really need. If we can't compensate $\boldsymbol{\varepsilon}_{d}$, the body must be in stable stage or we can't use it.

\section{B. Realization of Error Allocation}

Error allocation is realized by heading error allocation matrix $\mathbf{D}_{\text {yaw }}$ and horizontal attitude error allocation matrix $\mathbf{D}_{h}$.

where

$$
\mathbf{D}_{\text {yaw }}=\left[\begin{array}{lll}
\alpha_{x} & 0 & 0 \\
0 & \alpha_{y} & 0 \\
0 & 0 & \alpha_{z}
\end{array}\right], \mathbf{D}_{h}=\left[\begin{array}{lll}
\beta_{x} & 0 & 0 \\
0 & \beta_{y} & 0 \\
0 & 0 & \beta_{z}
\end{array}\right]
$$

Parameters in $\mathbf{D}_{\text {yaw }}$ and $\mathbf{D}_{h}$ have the following form of expression,

$$
\begin{aligned}
& \alpha_{x}=F_{1}\left(\left\|\mathbf{f}^{b}\right\|,\left|b_{y}\left(t_{k}\right)-b_{y}\left(t_{k-1}\right)\right|\right) \\
& \alpha_{y}=\alpha_{x}, \alpha_{z}=F_{2}\left(\left|b_{y}\left(t_{k}\right)-b_{y}\left(t_{k-1}\right)\right|\right) \\
& \beta_{x}=F_{3}\left(\left\|\mathbf{f}^{b}\right\|\right), \beta_{y}=\beta_{x} \\
& \beta_{z}=F_{4}\left(\left\|\mathbf{f}^{b}\right\|,\left|b_{y}\left(t_{k}\right)-b_{y}\left(t_{k-1}\right)\right|\right)
\end{aligned}
$$

Where $F_{1}(\|\bullet\|,|\bullet|), F_{2}(|\bullet|), F_{3}(\|\bullet\|)$ and $F_{4}(\|\bullet\|,|\bullet|)$ are regulation function.

For vector $\mathbf{x}$ and scalar ${ }^{y}$, there is

$$
F_{1}(\|\mathbf{x}\|,|y|)= \begin{cases}\|\mathbf{x}\| / \alpha_{t h 1}, & \|\mathbf{x}\|<\alpha_{t h 1} \&|y|<\alpha_{t h 2} \\ 1 & ,\|\mathbf{x}\|>\alpha_{t h 1} \&|y|<\alpha_{t h 2} \\ 0 & , \text { others }\end{cases}
$$

$$
F_{2}(|y|)= \begin{cases}1 & ,|y|<\alpha_{\text {th } 2} \\ 0, & \text { others }\end{cases}
$$

$$
F_{3}(\|\mathbf{x}\|)= \begin{cases}1 & ,\|\mathbf{x}\|<\alpha_{\text {th1 }} \\ 0 & , \text { others }\end{cases}
$$

$$
F_{4}(\|\mathbf{x}\|,|y|)= \begin{cases}|y| / \alpha_{t h 2} & ,|y|<\alpha_{t h 2} \&\|\mathbf{x}\|<\alpha_{t h 1} \\ 1 & ,|y|>\alpha_{t h 2} \&\|\mathbf{x}\|<\alpha_{t h 1} \\ 0 & , \text { others }\end{cases}
$$

$\alpha_{\text {th } 1 \text { and }} \alpha_{\text {th2 }}$ are all positive threshold.

From equation (4),

$$
\mathbf{e}=\mathbf{D}_{\text {yaw }} \cdot \boldsymbol{\varepsilon}_{\text {mag }}+\mathbf{D}_{h} \cdot \boldsymbol{\varepsilon}_{a c c}
$$


Rewriting the reference angle error $\mathbf{e}$ with diagonal matrix $\mathbf{D}_{\text {yaw }}$ and $\mathbf{D}_{h}$,

$$
\mathbf{e}=\left[\begin{array}{c}
\alpha_{x} \cdot \varepsilon_{\text {mag }, x} \\
\alpha_{y} \cdot \varepsilon_{\text {mag }, y} \\
\alpha_{z} \cdot \varepsilon_{m a g, z}
\end{array}\right]+\left[\begin{array}{c}
\beta_{x} \cdot \varepsilon_{a c c, x} \\
\beta_{y} \cdot \varepsilon_{a c c, y} \\
\beta_{z} \cdot \varepsilon_{a c c, z}
\end{array}\right]
$$

For equation (13), there is

$$
\mathbf{e}=\left[\begin{array}{l}
F_{1}(\bullet) \cdot \varepsilon_{\text {mag }, x} \\
F_{1}(\bullet) \cdot \varepsilon_{\text {mag }, y} \\
F_{2}(\bullet) \cdot \varepsilon_{\text {mag }, z}
\end{array}\right]+\left[\begin{array}{l}
F_{3}(\bullet) \cdot \varepsilon_{a c c, x} \\
F_{3}(\bullet) \cdot \varepsilon_{a c c, y} \\
F_{4}(\bullet) \cdot \varepsilon_{a c c, z}
\end{array}\right]
$$

When UAV is in fast angular motion, tangent acceleration take more important leading to $\left\|\mathbf{f}^{b}\right\|>\alpha_{\text {th1 } 1}$ and limited magnetic disturbance. The gravity reference error angle is not believable for its all three elements and limited believable for geomagnetic reference error angle, therefore reference angle error is $\mathbf{e}=\boldsymbol{\varepsilon}_{\text {mag }}$.

There exist other three using states for $\boldsymbol{\varepsilon}_{\text {mag }}$ and $\boldsymbol{\varepsilon}_{\text {acc }}$, listed as

1)limited magnetic disturbance \& limited tangent acceleration

$$
\left\|\mathbf{f}^{b}\right\|<\alpha_{t h 1} \&\left|b_{y}\left(t_{k}\right)-b_{y}\left(t_{k-1}\right)\right|<\alpha_{t h 2}
$$

2)large magnetic disturbance $\&$ limited tangent acceleration

$$
\left\|\mathbf{f}^{b}\right\|<\alpha_{t h 1} \&\left|b_{y}\left(t_{k}\right)-b_{y}\left(t_{k-1}\right)\right|>\alpha_{t h 2}
$$

3) large magnetic disturbance $\&$ large tangent acceleration

$$
\left\|\mathbf{f}^{b}\right\|>\alpha_{t h 1} \&\left|b_{y}\left(t_{k}\right)-b_{y}\left(t_{k-1}\right)\right|>\alpha_{t h 2}
$$

Especially for large magnetic disturbance \& large tangent acceleration, $\mathbf{e}=0$. So $\delta \boldsymbol{\omega}=0$ in equation (4).

\section{EXPERIMENTS RESULTS}

\section{A. Experiment Conditions}

MPU9250 in mobile phone which consists of 3 axis accelerometers, 3 axis gyroscopes and 3 axis magnetometer is provided for our experiment. The mobile phone is placed on wooden table that is far away from iron matter and is fixed to one direction with two pieces of plastic board. Initial heading is set to zero, and the positive axis direction in body frame fixed with mobile is right $\left({ }^{x}\right)$, forward $\left({ }^{y}\right)$ and up $\left({ }^{z}\right)$. Three stages are given in experimental, they are first stationary phase, motion phase and second stationary phase. First stage lasts 18 minutes, motion stage lasts 1.4 minutes, the third stage lasts 17 minutes. Sampling frequency is $50 \mathrm{~Hz}$. Data output of magnetometers is all available during three stages.

\section{B. Experiment Results}

For this experimental, proportion constant $k_{p}=0.2 \mathrm{rad} / \mathrm{s}$ and integral constant $k_{I}=0.015 \mathrm{rad} / \mathrm{s}$, acceleration threshold is $\alpha_{\text {th } 1}=13 \mathrm{~m} / \mathrm{s}^{2}$. Different filtering effects are shown in "Fig.3", including direct solutions, complementary filter without error allocation and complementary filter with error allocation.

"Fig.3” Comparison between direct solutions, complementary filter without error allocation and complementary filter with error allocation. Reference attitude is provided by accelerometer, during the first and third stage, attitude can be thought as reference attitude, attitude in second stage cannot be used as reference attitude because of accelerometer cannot provide believable information. Attitude with direct solution is poor to the results of complementary filter. Slowly attitude angle error increase. From the second stage to third stage, complementary filter make it work.
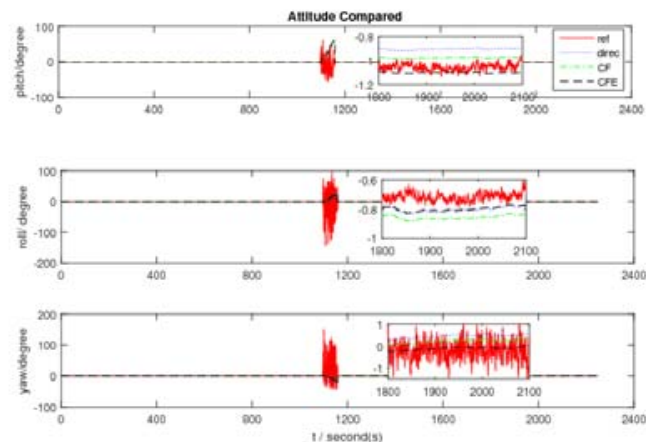

FIGURE III. COMPARISON BETWEEN DIRECT SOLUTIONS, COMPLEMENTARY FILTER WITHOUT ERROR ALLOCATION AND COMPLEMENTARY FILTER WITH ERROR ALLOCATION

Controller force the output signal of analog control module to track reference signal.

When AGC works in the state of two-phase flow, the intelligent controller plays a role of drive controller to supply the drive coil with the enough voltage, so that the tubes forced vibration. Thus, compared with analog-driven solutions, this analog-digital hybrid drive solution greatly improves the performance of the CMF allocation strategy especially in wide range of motion. During static motion, error allocation still has better effect. But the gyroscope bias is more time-varying with the error allocation for reason of violent modulus change of accelerometer. This is different than traditional complementary get smooth bias. 

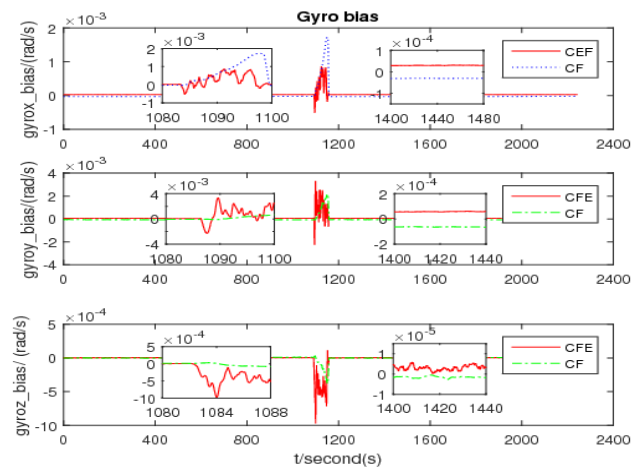

FIGURE IV. COMPARISON OF GYROSCOPE ESTIMATED BIAS BETWEEN COMPLEMENTARY FILTER WITHOUT ERROR ALLOCATION AND COMPLEMENTARY FILTER WITH ERROR ALLOCATION

\section{CONCLUSION}

The complementary filter is designed by error allocation strategy. With low-cost inertial measurement unit and magnetometer MPU9250 in mobile phone, the effectiveness of error allocation strategy is verified and is compared with traditional complementary.

\section{ACKNOWLEDGMENT}

This paper was supported by graduate seed fund (Z2017031) of North Western Polytechnical of Univercity (NWPU) for innovation and Entrepreneurship and supported by the key research and development plan(2017GY-044) , the technology plan (GX1701)of beilin government in xi'an, Shaanxi province.

\section{REFERENCES}

[1] A-J. Baerveldt and R. Klang. A low-cost and low-weight attitude estimation system for an autonomous helicopter. Intelligent Engineering Systems, 1997.

[2] M Euston, P Coote, R Mahony J Kim. A Complementary Filter for Attitude Estimation of a Fixed-Wing UAV. IEEE/RSJ International Conference on Intelligent Robots and Systems, 2008 340-345

[3] R Mahony, T Hamel, JM Pflimlin. Complementary filter design on the special orthogonal group SO(3). European Control Conference. CDCECC '05. 44th IEEE Conference on Decision and Control, 2005.

[4] D. Gebre-Egziabher, R.C. Hayward, and J.D. Powell. Design of multisensor attitude determination systems. IEEE Transactions on Aerospace and Electronic Systems, 40(2):627-649, April 2004.

[5] Y Oshiman A Carmi. Attitude Estimation from Vector Observations Using a Genetic-Algorithm-Embedded Quaternion Particle Filter. Journal of Guidance Control \& Dynamics, 2006, 29 (4): 879-891.

[6] Robert Mahony, Tarek Hamel, Pascal Morin. Nonlinear complementary filters on the special linear group.International Journal of Control, 2012, 85 (10): 1557-1573.

[7] P Marantos, Y Koveos, KJ Kyriakopoulos. UAV State Estimation Using Adaptive Complementary Filters. IEEE Transactions on Control Systems Technology, 2016, 24 (4): 1214-1226.

[8] L Benziane, AE Hadri, A Seba. Attitude Estimation and Control Using Linearlike Complementary Filters: Theory and Experiment. IEEE Transactions on Control Systems Technology, 2016, 24 (6): 2133-2140

[9] Z Wu, Z Sun, W Zhang, Q Chen. A Novel Approach for Attitude Estimation Based on MEMS Inertial Sensors Using Nonlinear Complementary Filters. IEEE Sensors Journal, 2016, 16 (10): 3856-3864
[10] C Coopmans, AM Jensen, Y Chen. Fractional-Order Complementary Filters for Small Unmanned Aerial System Navigation. 2013 International Conference on Unmanned Aircraft Systems (ICUAS), 2013, 73 (1-4): 754-760.

[11] F Sandhu, H Selamat, YM Sam. Non-Linear Complementary SO(3) Filters for Attitude Estimation and Navigation of Ground Vehicles. Applied Mechanics \& Materials, 2014, 663 (2): 139-152.

[12] JF Vasconcelos, B Cardeira, C Silvestre. Discrete-Time Complementary Filters for Attitude and Position Estimation: Design, Analysis and Experimental Validation. IEEE Transactions on Control Systems Technology, 2011, 19 (1): 181-198.

[13] JF Vasconcelos, C Silvestre, P Oliveira. Discrete time-varying attitude complementary filter. American Control Conference, 2009: 4056-4061.

[14] D Cao, Q Qu, C Li. Research of Attitude Estimation of UAV Based on Information Fusion of Complementary Filter. Fourth International Conference on Computer Sciences and Convergence Information Technology, 2009.

[15] PG Savage. Strapdown Inertial Navigation Integration Algorithm Design Part 2: Velocity and Position Algorithms. Journal of Guidance Control \& Dynamics, 1998, 21 (2): 208-221. 